\title{
Las gramíneas endémicas de Colombia
}

\section{The endemic grasses to Colombia}

\section{Diego Giraldo-Cañas*}

\section{RESUMEN}

Se presenta el inventario de las gramíneas endémicas de Colombia, con el objetivo de contribuir al conocimiento de la flora colombiana. Se realizaron numerosas expediciones de campo a diez de las once grandes regiones naturales de Colombia (Amazonia, Andes, Caribe, Chocó Biogeográfico, Guayana, islas caribeñas, isla de Malpelo, Orinoquia, Sierra Nevada de Santa Marta, valle del río Cauca y valle del río Magdalena). Además, se consultaron colecciones de los herbarios CAUP, COAH, COL, CUVC, CHOCO, HFAB, HUA, HUQ, INPA, JAUM, MEDEL, MEXU, MO, NY, PSO, RSA, SI, UDBC, UIS, US, VALLE y VEN, así como una revisión exhaustiva de literatura. Son endémicos de Colombia un género (Agrostopoa, Pooideae) y 77 especies. Así, el endemismo constituye 8,7\% de la flora agrostológica colombiana. Los géneros más ricos en especies endémicas son Festuca (17 especies), Chusquea (11), Andropogon (4), Arthrostylidium (4), Aulonenia (4) y Calamagrostis (4). La región andina es la más rica en especies endémicas (59 especies). Los gradientes altitudinales más ricos en especies endémicas son los de tierras altas (3000-5000 m), con cerca del 50\% de endemismo.

Palabras clave: Flora de Colombia; Gramíneas endémicas; Gramíneas neotropicales; Plantas endémicas; Poaceae de Colombia.

\begin{abstract}
The checklist of Colombian endemic grasses is given, in order to contribute to the knowledge of Colombian flora. This research is based on personal inventories in ten of the eleven greatest natural regions of Colombia (Amazon, Andes, Caribbean, Caribbean Islands, Malpelo Island, Chocó Biogeográfico, Guayana, Orinoquia, Sierra Nevada de Santa Marta, Cauca valley, and Magdalena valley), on the study of collections of different herbaria (CAUP, COAH, COL, CUVC, CHOCO, HFAB, HUA, HUQ, INPA, JAUM, MEDEL, MEXU, MO, NY, PSO, RSA, SI, UDBC, UIS, US, VALLE, and VEN), and on an exhaustive bibliographic research. One genus (Agrostopoa, Pooideae) and 77 species are endemic to Colombia. Thus, the endemism constitutes $8.7 \%$ of the Colombian agrostological flora. The genera with the high endemism are Festuca (17 species), Chusquea (11), Andropogon (4), Arthrostylidium (4), Aulonenia (4), and Calamagrostis (4). The Andean Region shows the highest endemism (59 species). The altitudinal gradients with more endemic species belonging to highlands (3000-5000 m), with ca. 50\% of endemism.
\end{abstract}

Keywords: Endemic grasses; Endemic plants; Flora of Colombia; Neotropical grasses; Poaceae of Colombia.
* Instituto de Ciencias Naturales, Facultad de Ciencias, Universidad Nacional de Colombia, Bogotá, Colombia. e-mail: dagiraldoc@unal.edu.co Recibido: 29 de marzo de 2012 Aceptado: 30 de abril de 2012 


\section{Bioetnia Volumen 9 № 1 (enero-junio), 2012}

\section{INTRODUCCIÓN}

En los estudios sobre biodiversidad, además de los análisis acerca de los patrones de riqueza y distribución de especies, entran en juego los análisis de endemismo, porque estos son de suma importancia en las estrategias de conservación de la naturaleza y manejo de la misma, toda vez que las especies endémicas no se distribuyen aleatoriamente (Kessler, Kluge 2008). Las especies endémicas están principalmente distribuidas en islas oceánicas y en hábitats insulares, aunque también pueden presentarse en hábitats aparentemente homogéneos como los bosques amazónicos o los bosques andinos húmedos (Kessler, Kluge 2008).

Así, el endemismo se define como la situación donde una especie, varias especies o un grupo taxonómico de especies (una familia, un género, un subgénero, etc.) están limitados a una región geográfica particular, debido a aislamiento, composición edáfica, topografía, clima o características ecológicas propias de los taxones (Kessler 2002, Morris, Morris 2003, Kessler, Kluge 2008), o estar restringidos a una región biogeográfica o incluso, a una división políticoadministrativa (p.e.un país, un departamento, una provincia, etc.). Por lo tanto, una especie endémica, un género endémico o una familia endémica se reconoce como aquella entidad biológica nativa y exclusiva de un ambiente, un área o una región geográfica particular (Morris, Morris 2003, De Carvalho 2011).

Según Font Quer (1993), existen tres clases de endemismo:

1. Endemismo conservativo o paleoendemismo (endemismo que se remonta a épocas geológicas antiguas).

2. Endemismo progresivo, neógeno o neoendemismo (endemismo de formación relativamente reciente).

3. Endemismo secundario (tratándose de un archipiélago, el endemismo de cada isla con respecto a las demás).

En este contexto, se presenta el inventario de las gramíneas endémicas de Colombia, con el objetivo de contribuir al conocimiento de la flora colombiana y como un insumo para investigaciones futuras que aborden la definición de áreas de endemismo en Colombia -desde la incorporación de datos de diferentes grupos vegetales y animales- con base en los planteamientos de De Carvalho (2011).

\section{MATERIALES Y MÉTODOS}

Expediciones de campo y muestreos. Se realizaron 104 viajes a diferentes localidades en diez de las once grandes regiones naturales de Colombia-Amazonia, Andes, Caribe, Chocó Biogeográfico, Guayana, islas caribeñas, isla de Malpelo (ésta es la única área en la que aún no se ha detectado la presencia de ninguna gramínea), Orinoquia, Sierra Nevada de Santa Marta, valle del río Cauca y valle del río Magdalenaentre los años 1995 y 2012, en las que se inventariaron los recursos agrostológicos, tanto en áreas naturales (conservadas y/o alteradas en diferentes etapas sucesionales) como en áreas rurales y urbanas. Los muestreos se realizaron en forma aleatoria y cualitativa y la recolección de ejemplares se ajustó a los estándares de inventarios florísticos y de preservación de las muestras (Johnston 1941, Lot, Chiang 1986, Gould, Shaw 1992, Llorente Bousquets et al. 1994). El inventario se complementó con el estudio de colecciones de los herbarios CAUP, COAH, COL, CUVC, CHOCO, HFAB, HUA, HUQ, INPA, JAUM, MEDEL, MEXU, MO, NY, PSO, RSA, SI, UDBC, UIS, US, VALLE y VEN -abreviados de acuerdo con Holmgren et al. (1990)- y adicionalmente, se consultaron numerosas fuentes bibliográficas.

Determinación taxonómica. Los ejemplares recolectados se determinaron taxonómicamente en el Herbario Nacional Colombiano (COL), en donde además se encuentran depositados con la serie de colección de D. Giraldo-Cañas. Todos los especímenes recolectados fueron determinados por el autor, aunque la Dra. María Negritto (Universidad del Magdalena, Santa Marta, Colombia) confirmó y/o corrigió algunas determinaciones taxonómicas de ejemplares del género Poa. Los nombres taxonómicos de todas las especies están basados en los recientes catálogos de las gramíneas del Nuevo Mundo (Judziewicz et al. 2000, Peterson et al. 2001, Soreng et al. 2003 y Zuloaga et al. 2003), excepto para los géneros Arthropogon (Giraldo-Cañas 2011a), Axonopus (Giraldo-Cañas 2001a,2008), Digitaria (Giraldo-Cañas 2005, 2010), Festuca (Stanèík 2003, Stanèík \& Peterson 2007), Glyceria (Giraldo-Cañas 2002, Tsvelev 2006) y Panicum (Zuloaga, Giraldo-Cañas 2012).

Intervalos altitudinales. En relación con la consideración de los intervalos altitudinales, hay que destacar que estos se discriminaron arbitrariamente con una amplitud de $500 \mathrm{~m}$, aunque esta elección se hizo con base en el uso frecuente de intervalos con esta amplitud en diferentes investigaciones en gradientes altitudinales (Giraldo-Cañas 2011b), lo cual facilita las comparaciones de riqueza, diversidad y endemismo entre diferentes grupos biológicos. Cabe destacar que en el último intervalo altitudinal (5000-5775 m) no se han encontrado gramíneas. Así, el límite de distribución altitudinal de las especies vegetales en Colombia se ubica entre los 4700 y los $4800 \mathrm{~m}$ en la región andina (GiraldoCañas, obs. pers.) y entre los 4800 y los 5000 (5100) m en la Sierra Nevada de Santa Marta (Cleef, Rangel Ch. 1984), lo que se ajusta a las características del efecto de masa o efecto de Massenerhebung(Flenley 2007), porque la Sierra Nevada de Santa Marta está más cerca del mar Caribe, donde tiene las influencias directas de las corrientes caribeñas, las cuales son más cálidas. La incorporación de un análisis por gradientes altitudinales, constituye una técnica eficaz para detectar los cambios en la dinámica, la estructura y el funcionamiento de los ecosistemas y así, esta aproximación permite entender 
cómo las poblaciones y las comunidades cambian en respuesta a las variaciones ambientales, ya sean bióticas o abióticas (Austin 1985, Gosz 1992, Sanders 2002). Es por esto que los datos aquí presentados, pretenden cubrir algunos vacíos en torno de estos aspectos ecológicos de la distribución de angiospermas neotropicales.

\section{RESULTADOS Y DISCUSIÓN}

Un género [Agrostopoa Davidse, Soreng, P. M. Peterson, Pooideae (Andes y Sierra Nevada de Santa Marta) (Davidse et al. 2009)] y 77 especies son endémicos de Colombia (Tabla 1, Anexo 1). La baja proporción de elementos endémicos $(8,7 \%$ del total de las especies de gramíneas presentes en Colombia) refuerza la hipótesis de que Colombia constituye un puente biogeográfico entre Mesoamérica y Sudamérica, en donde convergen biotas de diferente origen y por lo tanto, el endemismo es bajo a moderado en territorio colombiano, excepto en los páramos, en donde el porcentaje de endemismo del total de la flora vascular se ubica entre $45 \%$ y $63 \%$ (Rangel-Ch. 2000).

Así, en Colombia se han documentado 59 géneros endémicos de plantas vasculares, los cuales son representantes de 27 familias, entre las que se destacan las Asteraceae (nueve géneros endémicos), las Orchidaceae (8), las Melastomataceae (7) y las Rubiaceae (4) (Lozano Contreras 1996). Cabe destacar que la mayoría de estos géneros se concentra en la región andina y en la Sierra Nevada de Santa Marta (Lozano Contreras 1996).

La mayor cantidad de elementos agrostológicos endémicos se presenta en la región andina, con 59 especies, más dos especies que son compartidas con la Sierra Nevada de Santa Marta, y una especie compartida con la Orinoquia (Anexo 1). En las restantes regiones naturales el número de elementos endémicos no supera las siete especies: la Guayana colombiana tiene siete especies endémicas (más dos compartidas con la Amazonia, una compartida con la Orinoquia y una compartida con la Amazonia y la Orinoquia), Sierra Nevada de Santa Marta (tres especies endémicas), la Orinoquia (2), y el Chocó Biogeográfico (1). Cabe destacar que Carbonó, Lozano-Contreras (1997) no citaron especies endémicas de gramíneas para la Sierra Nevada de Santa Marta. En la Amazonia, la Llanura del Caribe, así como en las islas caribeñas y en los valles geográficos de los ríos Cauca y Magdalena no se han detectado especies endémicas (Anexo 1). Por otra parte, hay que destacar que los géneros que presentan el mayor número de especies endémicas son Festuca (Pooideae) con 17 especies endémicas, Chusquea (Bambusoideae, once especies endémicas), Arthrostylidium, Aulonemia (Bambusoideae), Andropogon (Panicoideae) y Calamagrostis (Pooideae) con cuatro especies endémicas cada uno (Tabla 1, Anexo 1).
Tabla 1

\section{Número de especies endémicas por género en la flora agrostológica colombiana}

\begin{tabular}{|c|c|}
\hline Género & Número de especies \\
\hline Festuca & 17 \\
\hline Chusquea & 11 \\
\hline Andropogon & 4 \\
\hline Arthrostylidium & 4 \\
\hline Aulonemia & 4 \\
\hline Calamagrostis & 4 \\
\hline Agrostis & 3 \\
\hline Agrostopoa* & 3 \\
\hline Digitaria & 3 \\
\hline Neurolepis & 3 \\
\hline Paspalum & 3 \\
\hline Axonopus & 2 \\
\hline Nassella & 2 \\
\hline Poa & 2 \\
\hline Rhipidocladum & 2 \\
\hline Arthropogon & 1 \\
\hline Cortaderia & 1 \\
\hline Glyceria & 1 \\
\hline Guadua & 1 \\
\hline Panicum & 1 \\
\hline Parodiolyra & 1 \\
\hline Raddiella & 1 \\
\hline Tripsacum & 1 \\
\hline Urochloa & 1 \\
\hline Zizaniopsis & 1 \\
\hline Total & 77 \\
\hline
\end{tabular}

* Único género endémico de gramíneas de Colombia.

Sólo se han encontrado cuatro trabajos que hablan del endemismo por región natural en Colombia: Betancur, Kress (1995) destacaron que el mayor porcentaje de las especies endémicas de la familia Heliconiaceae (73\%) se concentra en la región andina; Giraldo-Cañas (2011b) encontró que 72,7\% de las Marcgraviaceae endémicas se encuentran en la región andina, y Hernández Camacho et al. (1992a, 1992b), resaltaron que los mayores endemismos se concentran en la región andina -no obstante, estos últimos autores no proporcionan porcentajes de endemismo. Estos porcentajes son similares al que presenta la región andina para las gramíneas endémicas, el cual corresponde a 76,6\% de las 77 especies endémicas de Colombia (59 especies endémicas andinas). Cabe 


\section{Bioetnia Volumen 9 № 1 (enero-junio), 2012}

destacar que cerca del 50\% de las especies de gramíneas endémicas se encuentra en las tierras altas (3000-5000 m), ca. $29 \%$ en las tierras medias $(1500-3000 \mathrm{~m})$ y alrededor del $21 \%$ en las tierras bajas $(0-1500 \mathrm{~m})$ (Anexo 1$)$. Los anteriores datos se ajustan a los encontrados por otros autores (Carbonó, Lozano-Contreras 1997, Kessler 2002, Kessler, Kluge 2008), quienes resaltaron que el endemismo aumenta con la altitud en ambientes montanos tropicales [aunque esta afirmación depende del grupo biológico analizado (Kessler 2002)], mientras que la riqueza disminuye en un contexto de gradientes altitudinales, situación que se conoce con el nombre de Regla de Rapoport (Sanders 2002, Guerrero, Sarmiento 2010), lo cual se traduce en un alto recambio de las especies de gramíneas a medida que se aumenta el gradiente altitudinal (Giraldo-Cañas, obs. pers.).

Por último, es necesario destacar que las especies endémicas no han sido estudiadas en forma integral, las cuales, por sus características y rareza geográfica, pueden estar en peligo de extinción, y de las que poco se sabe acerca de sus características genéticas, demográficas y ecológicas, por lo que son necesarios estudios y análisis más precisos.

\section{AGRADECIMIENTOS}

Quiero manifestar mi profundo reconocimiento al Instituto de Ciencias Naturales de la Universidad Nacional de Colombia por todas las facilidades que me brindó para la preparación de este trabajo. A los curadores de los herbarios CAUP, COAH, COL, CUVC, CHOCO, HFAB, HUA, HUQ, INPA, JAUM, MEDEL, MEXU, MO, NY, PSO, RSA, SI, UDBC, UIS, US, VALLE y VEN por los préstamos enviados o por su grata colaboración durante la visita a sus instalaciones. A los herbarios de los jardines botánicos Rancho Santa Ana «RSA»(Claremont, California, EE.UU.) y Missouri «MO» (St. Louis, Missouri, EE.UU.), el Herbario Nacional de los EE.UU. («US», Smithsonian Institution, Washington DC), así como al Herbario del Instituto Darwinion «SI» (San Isidro, Buenos Aires, Argentina), por las facilidades económicas brindadas para las visitas a sus instalaciones. La Red Latinoamericana de Botánica (Santiago, Chile) otorgó las facilidades económicas para visitar el Herbario Nacional de México «MEXU»(UNAM, México DF, México) y el Herbario del Instituto de Botánica Darwinion. A la Dra. María Negritto (Universidad del Magdalena, Colombia) por la confirmación y/o corrección de algunas determinaciones taxonómicas de ejemplares del género Poa. A las diferentes personas de las numerosas áreas visitadas, por su valiosa colaboración, permanente amabilidad y por compartir con el autor todo su conocimiento en torno de la flora colombiana, especialmente a Jusset Sabagh, Elías Yukuna, Mayer Yukuna, Luis Tanimuka, Magola de Rúa, Ramón Nagles, Mireya Jaramillo, Marciano Córdoba, Inés de Montoya, Pablo Pala- cios. A los doctores Paul M. Peterson (US), Carlos Parra (COL), Maruja Uribe (CHOCÓ), Lady Vargas Porras (CHOCÓ), Fernando O. Zuloaga (SI), Robert Soreng (US), Steve A. Renvoize(KEW), Orlando Rivera (COL), Simon Lægaard (AAU) y Travis Columbus (RSA), por su valiosa y permanente colaboración, por el obsequio de valiosa bibliografía, así como por sus valiosos comentarios. Esta contribución se deriva del proyecto «Estudios morfológicos, anatómicos y taxonómicos en gramíneas neotropicales», de la Universidad Nacional de Colombia, Bogotá.

\section{LITERATURA CITADA}

Austin, M. P. 1985. Continuum concept, ordination methods, and niche theory. Ann Rev Ecol Syst. 16: 39-61.

Betancur, J., W. J. Kress. 1995. Distribución geográfica y altitudinal del género Heliconia (Heliconiaceae) en Colombia. En: S. P. Churchill, H. Balslev, E. Forero, J. L. Luteyn (eds.). Biodiversity and conservation of Neotropical montane forests. p. 513-23. Nueva York: The New York Botanical Garden. 702 pp.

Carbonó, E., G. Lozano-Contreras. 1997. Endemismos y otras singularidades de la Sierra Nevada de Santa Marta, Colombia. Posibles causas de origen y necesidad de conservarlos. Rev Acad Colomb Ci Exact. 21: 409-19.

Cavelier, J., D. Lizcaíno, M. T. Pulido. 2001. Colombia. En: M. Kappelle, A. D. Brown (eds.). Bosques nublados del neotrópico. p. 443-96. San José: Editorial INBio. 698 pp.

Cleef, A. M., O. Rangel Ch. 1984. La vegetación del páramo del noroeste de la Sierra Nevada de Santa Marta. En: T. van der Hammen, P.M. Ruiz (eds.). Estudios de ecosistemas tropandinos, la Sierra Nevada de Santa Marta (Colombia, transecto Buriticá-La Cumbre 2). p. 203-66. Berlín-Stuttgart: J. Cramer. 603 pp.

Davidse, G., R. J. Soreng, P. M. Peterson. 2009. Agrostopoa (Poaceae, Pooideae, Poeae, Poinae), a new genus with three species from Colombia. Novon. 19: 32-40.

De Carvalho, C. J. B. 2011. Áreas de endemismo. En: C. J. B. de Carvalho, E. A. B. Almeida (org.). Biogeografía da América do Sul. Padrões e processos. p. 41-51. São Paulo: Editora Roca Ltda. 306 pp.

Flenley, J. 2007. Ultraviolet insolation and the tropical rainforest: altitudinal variations, Quaternary and recent change, extinctions, and biodiversity. En: M. B. Bush, J. R. Flenley (eds.). Tropical rainforest responses to climatic change. p. 219-35. Berlin: Springer-Praxis Publishing. 396 pp.

Font Quer, P. 1993. Diccionario de botánica. Barcelona: Ed. Labor, S.A. 1244 pp.

Garzón, A., J. O. Rangel-Ch. 2004. Localidades del Chocó biogeográfico: ubicación y georreferenciación. En: J. O. Rangel Ch. (ed.). Colombia diversidad biótica IV. El Chocó Biogeográfico/Costa pacifica. p. 97785. Bogotá: Instituto de Ciencias Naturales, Universidad Nacional de Colombia. 997 pp.

Giraldo-Cañas, D. 2001a. Sinopsis de la sección Cabrera del género neotropical Axonopus (Poaceae: Panicoideae: Paniceae). Rev Acad Colomb Ci Exact. 25: 207-23.

Giraldo-Cañas, D. 2001b. Relaciones fitogeográficas de las sierras y afloramientos rocosos de la Guayana colombiana: un estudio preliminar. Rev Chil Hist Nat. 74: 353-64.

Giraldo-Cañas, D. 2002. Una nueva especie de Glyceria (Poaceae, Pooidea, Meliceae) de Colombia. Caldasia. 24: 9-13.

Giraldo-Cañas, D. 2005. Las especies colombianas del género Digitaria (Poaceae: Panicoideae: Paniceae). Caldasia. 27: 25-87.

Giraldo-Cañas, D. 2008. Sistemática del género Axonopus (Poaceae: Panicoideae: Paniceae) y revisión de las especies de la serie Barbigeri. Biblioteca José Jerónimo Triana. 17: 1-211.

Giraldo-Cañas, D. 2010. Una nueva especie de Digitaria (Poaceae: Panicoideae: Paniceae) de Colombia. Caldasia. 32: 301-9.

Giraldo-Cañas, D. 2011a. Una nueva especie de Arthropogon (Poaceae: Panicoideae: Paniceae) y primer registro del género en Colombia. Caldasia. 


\section{Las gramíneas endémicas de Colombia. D. Giraldo-Cañas}

33: $397-412$.

Giraldo-Cañas, D. 2011b. Las Marcgraviaceae de Colombia: inventario, diversidad, endemismo y distribución. Bioetnia. 8: 28-39.

Gosz, J. 1992. Gradient analysis of ecological change in time and space: implications for forest management. Ecol Appl. 2: 248-61.

Gould, F. W., R. B. Shaw. 1992. Gramíneas. Clasificación sistemática. México, D.F.: AGT Ed., S.A. 381 pp.

Guerrero, R. J., C. E. Sarmiento. 2010. Distribución altitudinal de hormigas (Hymenoptera, Formicidae) en la vertiente noroccidental de la Sierra Nevada de Santa Marta (Colombia). Acta Zool Mex. (nueva serie) 26: 279-302.

Hernández Camacho, J., R. Ortiz Quijano, T. Walschburger, A. Hurtado Guerra. 1992a. Estado de la biodiversidad en Colombia. Acta Zool Mex s.n. (edición especial): 41-3.

Hernández Camacho, J., A. Hurtado Guerra, R. Ortiz Quijano, T. Walschburger. 1992b. Centros de endemismo en Colombia. Acta Zool Mex. s.n. (edición especial): $175-90$.

Hernández Camacho, J., A. Hurtado Guerra, R. Ortiz Quijano, T. Walschburger. 1992c. Unidades biogeográficas de Colombia. Acta Zool Mex s.n. (edición especial): 105-51.

Holmgren, P., N. Holmgren, L. Barnett. 1990. Index Herbariorum. Part I: The Herbaria of the World. New York: The New York Botanical Garden. 693 pp.

Johnston, I. M. 1941. Preparación de ejemplares botánicos para herbario. Tucumán: Instituto Miguel Lillo, Universidad Nacional de Tucumán. 60 pp.

Judziewicz, E. J., R. J. Soreng, G. Davidse, P. M. Peterson, T. S. Filgueiras, F. O. Zuloaga. 2000. Catalogue of New World grasses (Poaceae): I. Subfamilies Anomochlooideae, Bambusoideae, Ehrhartoideae, and Pharoideae. Contr US Natl Herb. 39: 1-128.

Kessler, M. 2002. The elevational gradient of Andean plant endemism: varying influences of taxon-specific traits and topography at different taxonomic levels. J Biogeography. 29: 1159-65.

Kessler, M., J. Kluge. 2008. Diversity and endemism in tropical montane forests - from patterns to processes. Biodivers Ecol Series. 2: 35-50.

Llorente Bousquets, J., I. Luna Vega, J. Soberón Mainero, L. Bojórquez Tapia. 1994. Biodiversidad, su inventario y conservación: teoría y práctica en la taxonomía alfa contemporánea. En: J. Llorente Bousquets, I. Luna Vega (compiladores). Taxonomía biológica. p. 507-22. México, D.F.: Ediciones Científicas Universitarias, serie Texto Universitario, Universidad Nacional Autónoma de México-Fondo de Cultura Económica. 626 pp.

Lot, A., F. Chiang (compiladores). 1986. Manual de herbario. Administración $y$ manejo de colecciones, técnicas de recolección y preparación de ejemplares botánicos. México, D.F.: Consejo Nacional de la Flora de México. $142 \mathrm{pp}$.

Lozano Contreras, G. 1996. Géneros de cormofitos endémicos de Colombia. En: P. Pinto (ed.). Notas sobre biodiversidad. Biblioteca José Jerónimo Triana. 13: 65-71.

Morris, D. W., M. Z. Morris. 2003. English-Spanish dictionary of plant biology. Cambridge: Cambridge International Science Publishing. 647 pp.

Peterson, P. M., R. J. Soreng, G. Davidse, T. S. Filgueiras, F. O. Zuloaga, E. J. Judziewicz. 2001. Catalogue of New World grasses (Poaceae): II. Subfamily Chloridoideae. Contr US Natl Herb. 41: 1-255.

Rangel-Ch., J. O. 2000. Síntesis final: Visión integradora sobre la región del páramo. En: J. O. Rangel-Ch. (ed.). Colombia Diversidad Biótica III. La región de vida paramuna. p. 814-36. Bogotá, D.C.: Instituto de Ciencias Naturales, Universidad Nacional de Colombia. 902 pp.

Sanders, N. 2002. Elevational gradients in ant species richness: area, geometry, and Rapoport's rule. Ecography. 25: 25-32.

Soreng, R. J., P. M. Peterson, G. Davidse, E. J. Judziewicz, F. O. Zuloaga, T. S. Filgueiras, O. Morrone. 2003. Catalogue of New World grasses (Poaceae): IV. Subfamily Pooideae. Contr US Natl Herb. 48: 1-730.

Stanèík, D. 2003. Las especies del género Festuca (Poaceae) en Colombia. Darwiniana. 41: 93-153.

Stanèík, D., P. M. Peterson. 2007. A revision of Festuca (Poaceae: Loliinae) in South American paramos. Contr US Natl Herb. 56: 1-184.

Tsvelev, N. N. 2006. Synopsis of the mannagrass genus, Glyceria (Poaceae). Bot Zhurn. 91: 255-76.

Zuloaga, F. O, D. Giraldo-Cañas. 2012. Panicum (Poaceae: Panicoideae: Paniceae). Flora de Colombia (en imprenta).

Zuloaga, F. O., O. Morrone, G. Davidse, T. S. Filgueiras, P. M. Peterson, R. J. Soreng, E. J. Judziewicz. 2003. Catalogue of New World grasses (Poaceae): III. Subfamilies Panicoideae, Aristoideae, Arundinoideae, and Danthonioideae. Contr US Natl Herb. 46: 1-662.

\section{Anexo 1}

Inventario de las 77 especies endémicas de Poaceae de Colombia (sólo se ha documentado un género endémico: Agrostopoa Davidse, Soreng \& P. M. Peterson). La región o las regiones en las que crecen las especies se discriminaron así: Amazonia, Andes, Caribe, Chocó Biogeográfico, Guayana, islas caribeñas, isla de Malpelo [reconocida como una unidad biogeográfica independiente del Chocó Biogeográfico, de acuerdo con Hernández Camacho et al. (1992c)] (ésta es la única área en la que aún no se ha documentado la presencia de ninguna gramínea), Orinoquia, Sierra Nevada de Santa Marta, valle del río Cauca y valle del río Magdalena [cabe destacar que la serranía de La Macarena se incluyó en la región Guayana, de acuerdo con los criterios de Hernández Camacho et al. (1992c), Cavelier et al. (2001) y Giraldo-Cañas (2001b), mientras que la isla de Gorgona se incluyó en el Chocó Biogeográfico, de acuerdo con Garzón \& Rangel-Ch. (2004)]. Las siglas para los departamentos (Dept.) son las siguientes: AMA: Amazonas, ANT: Antioquia, ARA: Arauca, ATL: Atlántico, BOL: Bolívar, BOY: Boyacá, CAL: Caldas, CAQ: Caquetá, CAS: Casanare, CAU: Cauca, CES: Cesar, CHO: Chocó, COR: Córdoba, CUN: Cundinamarca, GUA: Guaviare, GUI: Guainía, GUJ: La Guajira, HUI: Huila, MAG: Magdalena, MET: Meta, NAR: Nariño, NSA: Norte de Santander, PUT: Putumayo, QUI: Quindío, RIS: Risaralda, SAN: Santander, SAP: San Andrés, Providencia y Santa Catalina, SUC: Sucre, TOL: Tolima, VAL: Valle del Cauca, VAU: Vaupés, VIC: Vichada. 
Agrostis boyacensis Swallen \& García-Barr.

Ref.: D. Giraldo-Cañas 5123 (COL).

Andes

3000-4500 m.

Dept.: BOY, CUN

\section{Agrostis lehmanni Swallen}

Ref.: E. Killip 38598 (US).

Andes

3000-4000 m.

Dept.: CAU

Agrostis scabrifolia Swallen

Ref.: D. Giraldo-Cañas 3982 (COL).

Andes

3000-3500 m.

Dept.: CAL, CUN, NSA

Agrostopoa barclayae Davidse, Soreng \&

P. M. Peterson

Ref.: H. Barclay \& P. Juajibioy 6567 (COL).

Sierra Nevada de Santa Marta

3000-3500 m.

Dept.: MAG

Agrostopoa wallisii (Mez) P. M. Peterson, Soreng

\& Davidse

Sin.: Muhlenbergia wallisii Mez

Ref.: H. Barclay 7079 (COL).

Sierra Nevada de Santa Marta

3500-4000 m.

Dept.: GUJ, MAG

Agrostopoa woodii Soreng, P. M. Peterson

\& Davidse

Ref.: J. Wood 5268 (COL).

Andes

4000-4500 m.

Dept.: BOY

\section{Andropogon bogotensis (Hack.) A. Zanin}

Sin.: Andropogon incanus Hack. var. bogotensis Hack.

Ref.: Karsten s.n. (US).

Orinoquia
0-500 m.

Dept.: MET

Andropogon lehmannii Pilg.

Ref.: F. C. Lehmann 6979 (US).

Andes

1500-3000 m.

Dept.: BOY, CAU, NAR, VAL

Andropogon tolimensis Pilg.

Ref.: F. C. Lehmann 4403-A (US).

Andes

1000-1500 m.

Dept.: CAU

\section{Andropogon sp. nov.}

Ref.: D. Giraldo-Cañas 4542 (COL).

Andes

1000-1500 m.

Dept.: NSA

Arthropogon sorengii Gir.-Cañas

Ref.: D. Giraldo-Cañas 4560 (COL).

Guayana

0-500 m.

Dept.: VAU

Arthrostylidium auriculatum Londoño \& L.G. Clark Ref.: X. Londoño \& L. Clark 895 (COL).

Andes.

1500-2000 m.

Dept.: ANT

\section{Arthrostylidium chiribiquetensis Londoño \&}

L.G. Clark

Ref.: P. Palacios et al. 2681 (COL).

Guayana

500-1000 m.

Dept.: CAQ

Arthrostylidium punctulatum Londoño \& L.G. Clark

Ref.: S. Díaz-Piedrahita 3399 (COL).

Andes

1500-2000 m. 
Dept.: SAN

Arthrostylidium virolinensis Londoño \& L.G. Clark

Ref.: S. Díaz-Piedrahita 4048 (COL).

Andes

1500-2000 m.

Dept.: SAN

Aulonemia bogotensis L.G. Clark

Ref.: D. Giraldo-Cañas 4412 (COL).

Andes

2500-4000 m.

Dept.: ANT, BOY, CUN

\section{Aulonemia pumila L.G. Clark \& Londoño}

Ref.: B. Ramírez 839 (COL).

Andes

2500-3500 m.

Dept.: CAU, NAR, PUT

\section{Aulonemia trianae (Munro) McClure}

Sin.: Arundinaria multiflora Döll, Arundinaria trianae Munro

Ref.: J. Cuatrecasas et al. 12624 (COL).

Andes.

2500-4000 m.

Dept.: ANT, BOY, CUN, HUI, NSA, SAN

Aulonemia ximenae L. G. Clark, Judz. \& Tyrell

Ref.: J. R. Wood 5064 (COL).

Andes

2500-4000 m.

Dept.: ANT, BOY, CUN, HUI, SAN

\section{Axonopus morronei Gir.-Cañas}

Ref.: D. Giraldo-Cañas 2624 (COL).

Guayana, Orinoquia

0-500 m.

Dept.: CAQ, GUV, MET

\section{Axonopus zuloagae Gir.-Cañas}

Ref.: D. Giraldo-Cañas $2588(\mathrm{COAH})$.

Guayana

0-500 m.

Dept.: AMA, CAQ, GUV

\section{Calamagrostis divergens Swallen}

Ref.: J. Cuatrecasas 12604-A (COL).

Andes

3000-3500 m.

Dept.: NSA

\section{Calamagrostis involuta Swallen}

Ref.: E. Killip 17618 (COL).

Andes

3500-4000 m.

Dept.: NSA, SAN

\section{Calamagrostis killipii Swallen}

Ref.: E. Killip 17466 (COL).

Andes

3500-4500 m.

Dept.: CUN, NSA, SAN

Calamagrostis pubescens (Pilg.) Pilg.

Sin.: Deyeuxia pubescens Pilg.

Ref.: Stübel 389-b (BAA).

Andes

3000-3500 m.

Dept.: NAR

Chusquea antioquensis L.G. Clark \& Londoño Ref.: L. Clark et al. 255 (COL).

Andes

2000-2500 m.

Dept.: ANT, CAL

Chusquea arachniforme L.G. Clark \& Londoño Ref.: R. Callejas et al. 8994 (HUA).

Andes

1500-2000 m.

Dept.: ANT

\section{Chusquea latifolia L.G. Clark}

Ref.: D. Giraldo-Cañas 4728 (COL).

Andes

1500-3000 m.

Dept.: ANT, CAL, CHO, CUN, QUI, RIS, TOL, VAL

\section{Chusquea ligulata Munro}




\section{Bioetnia Volumen 9 № 1 (enero-junio), 2012}

Ref.: M. Lindig 1125 (COL).

Andes

2000-2500 m.

Dept.: CUN

\section{Chusquea Iondoniae L.G. Clark}

Ref.: P. Franco 1487 (COL).

Andes

1500-3000 m.

Dept.: ANT, CAL, CHO, CUN, VAL

Chusquea longiprophylla L.G. Clark

Ref.: O. Haught 6132 (COL).

Andes

2000-3000 m.

Dept.: CUN, HUI, PUT, TOL

Chusquea purdieana Munro

Ref.: M. L. Grant 10726 (COL).

Andes, Sierra Nevada de Santa Marta

2000-3000 m.

Dept.: BOY, CES, CUR, MAG, NSA, SAN

\section{Chusquea sneidernii Aspl.}

Ref.: von Sneidern 1121 (NY).

Andes

2000-3000 m.

Dept.: CAU, VAL

Chusquea spadicea Pilg.

Ref.: D. Giraldo-Cañas 4794 (COL).

Andes

2500-4000 m.

Dept.: ANT, CAL, CUN, QUI

Chusquea spathacea McClure ex L.G. Clark

Ref.: O. Rangel et al. 11373-A (COL).

Andes

1500-2500 m.

Dept.: CES

\section{Chusquea tuberculosa Swallen}

Ref.: E. Killip 18005 (US).

Andes
2500-3000 m.

Dept.: SAN

Cortaderia columbiana (Pilg.) Pilg.

Sin.: Gynerium columbianum Pilg.

Ref.: D. Giraldo-Cañas 3247(COL).

Andes, Sierra Nevada de Santa Marta

2500-4000 m.

Dept.: ARA, BOY, CAU, CES, CUN, HUI, MAG, MET, NSA, SAN

Digitaria cardenasiana Gir.-Cañas

Ref.: D. Giraldo-Cañas 3556 (COL).

Andes, Orinoquia

0-2500 $\mathrm{m}$.

Dept.: BOY, MET, SAN, VIC

\section{Digitaria rangelii Gir.-Cañas}

Ref.: C. Fuentes \& J. Amaya 764 (COL).

Orinoquia

0-500 m.

Dept.: MET

Digitaria sp. nov.

Ref.: D. Giraldo-Cañas 3673 (COL).

Guayana

0-500 m.

Dept.: VIC

Festuca azucarica E.B. Alexeev

Ref.: D. Stanèik 3404 (COL).

Andes

3500-4000 m.

Dept.: CAL, CAU, CUN, QUI, RIS, TOL, VAL

\section{Festuca boyacensis Stanèík}

Ref.: D. Stanèik 2166 (COL).

Andes

3000-3500 m.

Dept.: BOY

Festuca chita Stanèík

Ref.: A. Etter 661 (COL).

Andes 
3000-3500 m.

Dept.: BOY

\section{Festuca chitagana Stanèík}

Ref.: X. Londoño 550 (COL).

Andes

3500-4500 m.

Dept.: ANT, BOY, SAN

Festuca cleefiana E.B. Alexeev

Ref.: A. Cleef 6826 (COL).

Andes

3000-4500 m.

Dept.: BOY, CUN, SAN

Festuca cocuyana Stanèík

Ref.: A. Cleef 9078 (COL).

Andes

3500-4500 m.

Dept.: ARA, BOY

Festuca colombiana E.B. Alexeev

Ref.: D. Giraldo-Cañas 3269 (COL).

Andes

3000-4000 m.

Dept.: BOY, CUN, MET, SAN

Festuca cundinamarcae E.B. Alexeev

Ref.: J. Cuatrecasas 7966 (COL).

Andes

2500-4000 m.

Dept.: BOY, CUN, MET

\section{Festuca hatico Stanèík}

Ref.: D. Stanèik 4290 (COL).

Andes

3500-4000 m.

Dept.: SAN

\section{Festuca monguensis Stanèík}

Ref.: D. Stanèik 2026 (COL).

Andes

3000-4000 m.

Dept.: BOY
Festuca nereidaensis Stanèík

Ref.: D. Stanèik 3401-B (COL).

Andes

3500-4000 m.

Dept.: CAL

\section{Festuca pilar-franceii Stanèík}

Ref.: D. Giraldo-Cañas 5025 (COL).

Andes

3000-4000 m.

Dept.: CUN, MET

\section{Festuca reclinata Swallen}

Ref.: J. Cuatrecasas 9970 (COL).

Andes

3500-4000 m.

Dept.: SAN

Festuca sanctae-marthae Stanèík

Ref.: J. Cuatrecasas 24532 (COL).

Sierra Nevada de Santa Marta

3500-4500 m.

Dept.: MAG

Festuca sumapana Stanèík

Ref.: A. Cleef 7930 (COL).

Andes

4000-4500 m.

Dept.: MET

\section{Festuca toca Stanèík}

Ref.: J. Wood 4481 (COL).

Andes

2500-4000 m.

Dept.: BOY, NSA

Festuca woodii Stanèík

Ref.: J. Wood 5254 (COL).

Andes

2500-4000 m.

Dept.: BOY, CUN

Glyceria colombiana Gir.-Cañas

Ref.: D. Giraldo-Cañas 3216 (COL). 
Bioetnia Volumen 9 No 1 (enero-junio), 2012

Andes

1500-2000 m.

Dept.: CUN

\section{Guadua incana Londoño}

Ref.: X. Londoño 214 (COL)

Andes

500-1000 m.

Dept.: CAQ, PUT

Nassella karstenii (Hitchc.) Peñailillo

Sin.: Stipa karstenii Hitchc.

Ref.: H. Karsten s.n. (US).

Andes

2500-3500 m.

Dept.: CUN

Nassella pittieri (Hitchc.) Peñailillo

Sin.: Stipa pittieri Hitchc.

Ref.: H. Pittier 1438 (US).

Andes

3000-3500 m.

Dept.: CAU, PUT

Neurolepis petiolata Davidse \& L.G. Clark

Ref.: J. L. Zarucchi et al. 6265 (COL).

Andes

2000-3500 m.

Dept.: ANT, CAL, CUN, PUT

Neurolepis silverstonei Davidse \& L.G. Clark

Ref.: F. A. Silverstone-Sopkin et al. 4409 (MO).

Andes

2500-3000 m.

Dept.: $\mathrm{CHO}$

Neurolepis tesselata (Pilg.) Pilg.

Sin.: Planotia tesselata Pilg.

Ref.: A. Stübel 410 (US).

Andes

3500-4000 m.

Dept.: CAU

Panicum petilum Swallen
Ref.: C. Grass/ 10009 (US).

Amazonia, Guayana, Orinoquia

0-500 m.

Dept.: MET, VAU

Parodiolyra colombiensis Davidse \& Zuloaga

Ref.: D. Restrepo 467(COAH).

Guayana

0-500 m.

Dept.: CAQ

\section{Paspalum hirtum Kunth}

Ref.: D. Giraldo-Cañas 3755 (COL).

Andes

2500-4000 m.

Dept.: ANT, BOY, CAL, CAU, CUN, HUI, NAR, TOL, VAL

\section{Paspalum petrosum Swallen}

Ref.: C. Grass/ 10015 (US).

Amazonia, Guayana

0-500 m.

Dept.: VAU

\section{Paspalum schultesii Swallen}

Ref.: R. E. Schultes 19934 (US).

Amazonia, Guayana

0-500 m.

Dept.: VAU

\section{Poa orthophylla Pilg.}

Ref.: D. Giraldo-Cañas 3278 (COL).

Andes

3000-5000 m.

Dept.: BOY, CAL, CUN, MET, NAR, NSA

Poa soderstromii Negritto \& Anton

Ref.: T. Soderstrom 1310 (COL).

Andes

3500-4500 m.

Dept.: CAL, CUN

Raddiella molliculma (Swallen) C.E. Calderón \& Soderstr.

Sin.: Panicum molliculmum Swallen 
Ref.: R. E. Schultes 5662-A (COL).

Guayana

0-500 m.

Dept.: CAQ

Rhipidocladum abregoensis Londoño \& L.G. Clark Ref.: X. Londono et al. 881 (COL).

Andes

1000-2000 m.

Dept.: NSA

\section{Rhipidocladum Iongispiculatum Londoño \&}

L.G. Clark

Ref.: J. Betancur 4169 (COL).

Andes

2000-3000 m.

Dept.: ANT, BOY, CUN, NSA
Tripsacum cundinamarcae de Wet

Ref.: V. Patiño 65-3 (CEL).

Andes

1000-1500 m.

Dept.: CUN

Urochloa albicoma (Swallen \& García-Barr.) Morrone \& Zuloaga

Sin.: Panicum albicomum Swallen \& García-Barr. Ref.: F. Pennell 8129 (US).

Andes

1500-2000 m.

Dept.: CAU

\section{Zizaniopsis killipii Swallen}

Ref.: E. Killip 39099 (COL).

Chocó Biogeográfico

0-500 m.

Dept.: $\mathrm{CHO}$ 